\title{
Analytical Analysis of Single-Photon Correlations Emitted by Disordered Semiconductor Heterostructures
}

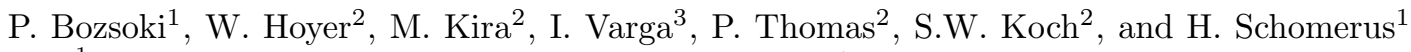 \\ ${ }^{1}$ Department of Physics, Lancaster University, UK-LA1 $4 Y B$ Lancaster, United Kingdom \\ ${ }^{2}$ Department of Physics and Material Sciences Center, \\ Philipps-Universität Marburg, D-35032 Marburg, Germany and \\ ${ }^{3}$ Elméleti Fizika Tanszék, Fizikai Intézet, Budapesti Müszaki és \\ Gazdaságtudományi Egyetem, Budafoki út 8, H-1111 Budapest, Hungary
}

(Dated: July 23, 2021)

\begin{abstract}
In a recent publication [Phys. Rev. Lett. 97, 227402 (2006), arXiv: cond-mat/0611411], it has been demonstrated numerically that a long-range disorder potential in semiconductor quantum wells can be reconstructed reliably via single-photon interferometry of spontaneously emitted light.

In the present paper, a simplified analytical model of independent two-level systems is presented in order to study the reconstruction procedure in more detail. With the help of this model, the measured photon correlations can be calculated analytically and the influence of parameters such as the disorder length scale, the wavelength of the used light, or the spotsize can be investigated systematically. Furthermore, the relation between the proposed angle-resolved single-photon correlations and the disorder potential can be understood and the measured signal is expected to be closely related to the characteristic strength and length scale of the disorder.
\end{abstract}

PACS numbers: 78.55.-m, 42.50.-p, 71.35.-y, 78.30.Ly

\section{INTRODUCTION}

Disorder in semiconductor heterostructures has strong influence on their optoelectronic properties. Independently of whether disorder is introduced by design or due to random interface roughness or compositional fluctuations it can significantly alter the properties of the heterostructure $[1,2,3,4,5,6$, 7]. Recent publications show that long-range spatial disorder is an important topic from both the technological and the fundamental point of view $[5,8,9,10]$.

In order to enhance our understanding of the role of disorder in these systems, we have recently proposed an experimental scheme [11] which can be viewed as the Fourier analogue of nano- or microluminescence $[12,13,14,15]$. This scheme is based on measuring angular correlations of spontaneously emitted light and has been shown to give direct access to the spatial distribution of the optically active electronic states and to the effect of disorder on them. As our numerical investigations have shown the spatial distribution can be recovered very reliably via a Fourier transformation of the experimentally measurable photonic correlation [11].

In the present paper we present an extended analytical study using a simplified model of uncoupled two-level systems. The additional simplification introduced by neglecting the many-body interactions allows us to deepen our understanding and present the fundamental principles behind the suggested reconstruction procedure in a more transparent way. One of the aims is to get a more intuitive understanding of the measured correlation function, such that a basic understanding of the characteristic pa- rameters of the disorder landscape can be gained from the direct measurement results without any reconstruction procedure.

The paper is structured as follows: We describe the experimental setup, introduce the important photon-correlation functions, and outline the reconstruction procedure in Sec. II. In Sec. III we present general analytical results for a system of noninteracting two-level systems where the disorder appears as a varying transition energy from site to site. Finally, we choose a sinusoidal model potential instead of a truly random disorder potential. For this special case, further analytical results are derived in Sec. IV before we conclude.

\section{MEASURING CORRELATIONS}

Our suggested scheme is based on the angular photonic correlations of spontaneously emitted light. Previous calculations have shown that not only photon numbers $\left\langle B_{\mathbf{q}}^{\dagger} B_{\mathbf{q}}\right\rangle$, but also single-photon correlations of the form $\left\langle B_{\mathbf{q}}^{\dagger} B_{\mathbf{q}^{\prime}}\right\rangle$ between photons of different modes $\left(\mathbf{q} \neq \mathbf{q}^{\prime}\right)$ are building up when a semiconductor heterostructure reaches quasi-equilibrium and spontaneously emits light [16]. While the rate of photons $\frac{\partial}{\partial t}\left\langle B_{\mathbf{q}}^{\dagger} B_{\mathbf{q}}\right\rangle$ is proportional to the photoluminescence $(\mathrm{PL})$ spectrum at energy $\omega_{q}=c|\mathbf{q}|$ and thus directly accessible to experiment [16, 17], a clever setup must be used in order to measure the complex correlations. The simplest possibility is to combine the light propagating along two different directions in a common detector. Here, the light emitted into directions $\mathbf{q}$ and $\mathbf{q}^{\prime}$ is redirected with the help of mirrors and through a beam splitter 
combined into the same detector. Consequently, the detector detects the combined beam and the correct detector operator for the description of the measurement process is given by

$$
d_{\mathbf{q}, \mathbf{q}^{\prime}}=B_{\mathbf{q}}+\mathrm{e}^{i \phi} B_{\mathbf{q}^{\prime}}
$$

i.e. a superposition of the photon operators of the two directions. The variable phase $\phi$ is adjusted via the optical path length difference between the two light beams. The detected signal is proportional to the number operator corresponding to $d_{\mathbf{q}, \mathbf{q}^{\prime}}$ and thus given by

$$
\begin{array}{r}
\left\langle d_{\mathbf{q}, \mathbf{q}^{\prime}}^{\dagger} d_{\mathbf{q}, \mathbf{q}^{\prime}}\right\rangle=\left\langle\left(B_{\mathbf{q}}^{\dagger}+\mathrm{e}^{-i \phi} B_{\mathbf{q}^{\prime}}^{\dagger}\right)\left(B_{\mathbf{q}}+\mathrm{e}^{i \phi} B_{\mathbf{q}^{\prime}}\right)\right\rangle \\
=\left\langle B_{\mathbf{q}}^{\dagger} B_{\mathbf{q}}\right\rangle+\left\langle B_{\mathbf{q}^{\prime}}^{\dagger} B_{\mathbf{q}^{\prime}}\right\rangle+2 \operatorname{Re}\left\langle\mathrm{e}^{i \phi} B_{\mathbf{q}^{\dagger}}^{\dagger} B_{\mathbf{q}^{\prime}}\right\rangle
\end{array}
$$

The first two terms on the right hand side of Eq. (2) are nothing but the separate PL spectra emitted into the two different directions while the interference term shows that also photon-correlations between the two different emission directions can be made visible. As previous results have shown, correlations can only build up if $\mathbf{q}_{\|}=\mathbf{q}_{\|}^{\prime}$ in the case of perfectly ordered system [18]. Here the parallel subscript denotes the component of the wave vector along the quantum well $(\mathrm{QW})$.

While the measured signal according to Eq. (2) still depends on a total of six components of $\mathbf{q}$ and $\mathbf{q}^{\prime}$, a few simplifications can be introduced according to the actual experiment; firstly, a spectrometer is used in order to achieve a spectrally and angularly resolved signal. The spectrometer is introduced after recombining the two emission directions into one beam and before the detector. Therefore, only the signal for equal $|\mathbf{q}|=\left|\mathbf{q}^{\prime}\right|$ has to be computed. Furthermore, our previous numerical investigations have shown that the interference depends much stronger on the difference $\mathbf{q}_{\|}-\mathbf{q}_{\|}^{\prime}$ than on the average value such that we define the angular correlation

$$
U_{\hbar \omega}\left(\Delta \mathbf{q}_{\|}\right)=\left\langle B_{\mathbf{q}^{\dagger}} B_{\mathbf{q}^{\prime}}\right\rangle
$$

with the specific choice of $\mathbf{q}^{\prime}=\left(\mathbf{q}_{\perp},-\Delta \mathbf{q}_{\|} / 2\right)$ and $\mathbf{q}=\left(\mathbf{q}_{\perp}, \Delta \mathbf{q}_{\|} / 2\right)$ as the key observable. The magnitude of the perpendicular component of $\mathbf{q}$ and $\mathbf{q}^{\prime}$ has to be fixed for each choice of $\Delta \mathbf{q}_{\|}$according to $\omega^{2}=c^{2}\left(\left|\mathbf{q}_{\perp}\right|^{2}+\left|\Delta \mathbf{q}_{\|}\right|^{2} / 4\right)$. Consequently, $U_{\hbar \omega}\left(\Delta \mathbf{q}_{\|}\right)$ depends on only two parameters, which is sufficient for a spectrally and angularly resolved scan.

While the magnitude of $\mathbf{q}_{\perp}$ is determined by $\omega$ and $\Delta \mathbf{q}_{\|}$, the sign can still be chosen differently. Our above choice of identical signs for the perpendicular components of $\mathbf{q}$ and $\mathbf{q}^{\prime}$ represents measurement in e.g. reflection geometry as depicted in Fig. 1 which has the advantage that non-transparent substrate can be used as both emission paths are on the

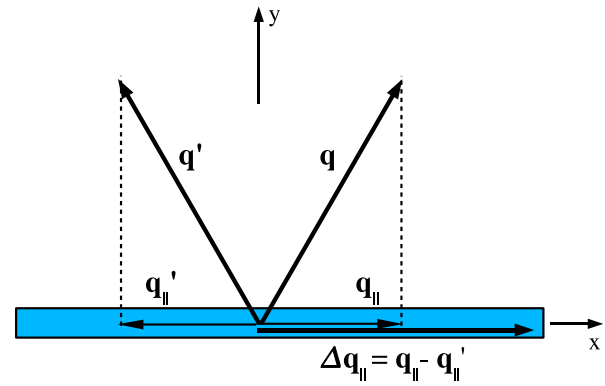

FIG. 1: Schematic setup of a possible experimental arrangement for measuring photonic correlations.

same side of the sample. An alternative possibility is to use light emitted into opposite directions which corresponds to $\mathbf{q}^{\prime}=-\mathbf{q}$. This setup was successfully applied to detect angular photonic correlations in ordered systems [18].

The principle idea behind our scheme for the reconstruction of the disorder potential exploits the fact that the strict momentum conservation along the direction parallel to the QW is broken by a disorder potential. In contrast to the ordered case where interference could only be observed for $\Delta \mathbf{q}_{\|}=0$, we expect that non-zero correlations cannot only be observed for vanishing $\Delta \mathbf{q}_{\|}$but also for other values. Please note that no such restriction applies to the direction perpendicular to the QW, independently if it is perfectly ordered or disordered, because the translational invariance is broken by the electronic confinement.

\section{A. Reconstruction procedure}

For the sake of simplicity of the analytical calculations we consider a one-dimensional quantum-wire in the remainder of the paper. In this case, the parallel component $q_{\|}$and the spatial coordinate $x$ along the wire are scalars. Apparently, all conclusions about momentum conservation hold as for a QW. To simplify the notation we use $\Delta q$ instead of $\Delta \mathbf{q}_{\|}$in the rest of the present work. During the calculations we limit ourselves to two emission directions with both planes of emission (spanned by the one-dimensional wire and the emission direction) being identical.

According to Eq. (2), the correlation function from Eq. (3) can be experimentally measured as an interference contrast [11]. By varying the optical path length $\phi$ in a controlled way, it is possible to extract both real and imaginary part of $U_{\hbar \omega}(\Delta q)$ such that it is legitimate to study the full complex value of $U_{\hbar \omega}(\Delta q)$.

By Fourier transforming the measured signal ac- 
cording to

$$
U_{\hbar \omega}(x)=\frac{1}{2 \pi} \int_{-\Delta q_{\max }}^{\Delta q_{\max }} U_{\hbar \omega}(\Delta q) e^{-i \Delta q x} \mathrm{~d} \Delta q
$$

one can obtain information on the spatial distribution of emitting sources of energy $\hbar \omega$. Here $\Delta q_{\max }$ is determined by the optical cone; since our scheme is a far-field method, the maximum value of $\Delta q$ accessible in an experiment is obtained for oblique incidence and given by approximately $\Delta q_{\max }=2 \omega_{0} / c=$ $4 \pi / \lambda_{0}$, where $c$ is the speed of light in vacuum and $\lambda_{0}$ and $\omega_{0}$ are the wavelength and frequency of the average emission frequency.

For a fixed value of $\omega$, Eq. (4) can exhibit multiple peaks at all those positions where the band gap corresponds to $\hbar \omega$ [11]. Thus, $U_{\hbar \omega}(x)$ can be viewed as a probability distribution of the local contributions to the emission spectrum. For a fixed value of $x$, on the other hand, $U_{\hbar \omega}(x)$ has only a single peak at the local transition energy such that the average energy can be defined as the weighted mean of the transition energies via

$$
U(x)=\int_{E_{1}}^{E_{2}} \hbar \omega U_{\hbar \omega}(x) \mathrm{d} \hbar \omega / \int_{E_{1}}^{E_{2}} U_{\hbar \omega}(x) \mathrm{d} \hbar \omega .
$$

Here, $E_{1}$ and $E_{2}$ are the limits of the energy scan carried out in the experiment. $U(x)$ is called the reconstructed disorder potential [11].

It is important to realize that the reconstruction procedure can only work within certain limits. Naturally, no disorder varying on length scales larger than the spot size can be detected. Furthermore, as with every far-field method, we are limited by the wavelength of the emission and the reconstructed disorder potential can only be determined if it varies on a typical length scale larger than the wavelenght of light. In the present paper we demonstrate this scheme on the basis of a theoretically calculated $U_{\hbar \omega}(\Delta q)$ for an ensemble of two-level systems.

\section{CORRELATIONS OF TWO-LEVEL SYSTEMS}

As starting point we consider an ensemble of uncoupled two-level systems as our model system. All two-level systems are spaced equally along $x$ with lattice constant $a$ such that the $j^{\text {th }}$ two-level system is positioned at the lattice site $R_{j}=a j$. By using an equation-of-motion approach and evaluating the Heisenberg equation of motion [11], we have shown previously that the correlation function can be expressed as

$$
U_{\hbar \omega}(\Delta q)=\frac{2}{\hbar}\left|\mathcal{F}_{\omega}\right|^{2} \sum_{j} \frac{\gamma S_{j} e^{i \Delta q R_{j}}}{\left(E_{j}-\hbar \omega\right)^{2}+\gamma^{2}} .
$$

Here, the prefactor contains the matrix element $\mathcal{F}_{\omega}$ of the light-matter coupling including the microscopic interband dipole matrix element, the so-called vacuum field amplitude, and the mode strength. The remaining term is a sum over sites with homogeneously broadened peaks centered at the site transition energies $E_{j}$ multiplied by the PL sources $S_{j}=f_{j}^{e} f_{j}^{h}$, where $f_{j}^{e}$ and $f_{j}^{h}$ are the electron and hole occupation probabilities of the respective site. The phase factor is a consequence of the different emission directions and is the key ingredient for observing the angle-resolved interference effects.

Taking the continuum limit $a \rightarrow 0$ with constant PL source density $S_{j} / a \rightarrow S(x)$ and a continuously changing transition energy $E(x)$, we can rewrite Eq. (6) as

$$
U_{\hbar \omega}(\Delta q)=\frac{2}{\hbar}\left|\mathcal{F}_{\omega}\right|^{2} \int \frac{\gamma e^{i \Delta q x} S(x)}{(E(x)-\hbar \omega)^{2}+\gamma^{2}} \mathrm{~d} x .
$$

In the ordered case, all transition energies $E(x)=$ $E_{0}$ are equal such that the angular correlation is proportional to a single broadened Lorentzian multiplied by the Fourier transform of the spot, $\int e^{i \Delta q x} S(x) \mathrm{d} x$.

\section{A. Spatial information}

According to Eq. (4), the Fourier transform of the correlation function gives some spatial information about the position of emitters of a certain frequency $\omega$. Starting with the result of Eq. (7), we obtain the Fourier transform

$$
\begin{array}{r}
U_{\hbar \omega}(x)=\frac{2\left|\mathcal{F}_{\omega}\right|^{2} \Delta q_{\max }}{\hbar} \int g_{\gamma}(E(y)-\hbar \omega) \\
S(y) \operatorname{sinc}\left(\Delta q_{\max }(y-x)\right) \mathrm{d} y(8)
\end{array}
$$

where we introduced the broadened $\delta$-function

$$
g_{\gamma}(E)=\frac{1}{\pi} \frac{\gamma}{E^{2}+\gamma^{2}}
$$

and used the sine-cardinal $\operatorname{sinc}(x)=\sin (x) / x$. The broadening of $g_{\gamma}$ is caused by the homogeneous line width $\gamma$ and results in a finite energy resolution. But also $\Delta q_{\max } \operatorname{sinc}\left(\Delta q_{\max } x\right)$ approaches a Dirac delta function for large values of $\Delta q_{\max }$. In that case, the broadening of the sinc function for finite values of $\Delta q_{\max }$ reflects the fact that no spatial information can be obtained for variations of the site energies on length scales below the wavelength $\lambda_{0}=4 \pi / \Delta q_{\max }$.

\section{B. Dimensionless parameters}

As one can anticipate from Eq. (8), the possibility to obtain precise spatial information strongly depends on the ratio $\gamma / W$ between homogeneous $\gamma$ and 
the disorder strength and on the ratios $\lambda_{0} / L$ and $\mathcal{S} / L$ between the observed wavelength or the spot size $\mathcal{S}$ and the typical disorder length scale $L$.

In order to obtain scaled equations with respect to the characteristic parameters $W$ and $L$ of the disorder potential, we introduce $u=x / L$ and $Q=$ $L \Delta q$ as new spatial and momentum coordinates and $\Omega=\hbar \omega / W$ and $\alpha=\gamma / W$ as the rescaled frequency and homogeneous line width. The maximum value of $u$ is determined by the spotsize to be $|u|<u_{\max }=\mathcal{S} / 2 L$ while $Q$ is confined according to $|Q|<Q_{\max }=\frac{4 \pi L}{\lambda_{0}}$. The dimensionless transition energy is denoted $\tilde{E}(x)=E(x) / W$. With the help of these new variables, we can rewrite Eqs. (6) and (8) as

$$
\begin{array}{r}
\tilde{U}_{\Omega}(Q)=\int g_{\alpha}(\tilde{E}(u)-\Omega) e^{i Q u} S(u) \mathrm{d} u \\
\tilde{U}_{\Omega}(u)=\frac{1}{\pi} \frac{Q_{\max }}{L} \int g_{\alpha}(\tilde{E}(v)-\Omega) S(v) \\
\operatorname{sinc}\left(Q_{\max }(v-u)\right) \mathrm{d} v
\end{array}
$$

where we have eliminated the prefactors by the definition $U_{\hbar \omega}=2 \pi L\left|\mathcal{F}_{\omega}\right|^{2} /(\hbar W) \tilde{U}_{\Omega}$ and $g_{\alpha}(\tilde{E})=$ $\pi^{-1} \alpha /\left(\left(\tilde{E}^{2}+\alpha^{2}\right)=W g_{\gamma}(E)\right.$. By virtue of the definition of $\tilde{E}$, its fluctuation is on the order of unity and the energy resolution can be directly estimated from the magnitude of $\alpha$. In the limit of strong disorder $(W \gg \gamma)$ corresponding to $\alpha \rightarrow 0$ the broadened $g_{\alpha}$ becomes a sharp Dirac delta function and we obtain

$$
\begin{gathered}
\left.\tilde{U}_{\Omega}(Q)\right|_{W \gg \gamma}=\sum_{n} e^{i Q u_{n}} S\left(u_{n}\right)\left(\left.\frac{\mathrm{d} \tilde{E}}{\mathrm{~d} u}\right|_{u=u_{n}(\Omega)}\right)^{-1} \\
\left.\tilde{U}_{\Omega}(u)\right|_{W \gg \gamma}=\frac{1}{\pi} \frac{Q_{\max }}{L} \sum_{n} \operatorname{sinc}\left(Q_{\max }\left(u_{n}-u\right)\right) \\
S\left(u_{n}\right)\left(\left.\frac{\mathrm{d} \tilde{E}}{\mathrm{~d} u}\right|_{u=u_{n}(\Omega)}\right)^{-1}
\end{gathered}
$$

where in both equations the sum over $n$ runs over all roots $u_{n}=u_{n}(\Omega)$ of the argument of the delta function. These roots are defined via $\tilde{E}\left(u_{n}(\Omega)\right)=\Omega$. Obviously, only positions $u_{n}$ within the spot can contribute to the correlations, as is guaranteed by the factor $S\left(u_{n}\right)$ which vanishes outside the excitation spot.

Another interesting limit is obtained for a disorder length scale well above the wavelength of the emitted light, but still below the spotsize. In that limit $Q_{\max } \gg 1$ such that the sine cardinal function can be treated as a sharp delta function and we find

$$
\left.\tilde{U}_{\Omega}(u)\right|_{L \gg \lambda_{0}}=\frac{1}{\pi} \frac{Q_{\max }}{L} g_{\alpha}(\tilde{E}(u)-\Omega) S(u) .
$$

In that case, the spatial resolution is very good even for relatively large values of $\gamma$ since for fixed $u=$ $x / L$ the correlation function $U_{\Omega}(u)$ has its maximum at the transition energy $\hbar \omega=E(x)$. According to Eq. (5) the reconstruced potential follows exactly the actual energetic variation $E(x)$.

\section{MODEL POTENTIAL}

While the results of the previous section are valid for a periodic arrangement of non-interacting twolevel systems with arbitrary disorder (i.e. arbitrary variation of the transition energies), we will introduce additional approximations in the present section in order to simplify the equations even further. In particular, we study the interpretation of $\tilde{U}_{\Omega}(Q)$, i.e., the direct measurement signal. That way, we can see what information on the disorder potential can be gained from the measurement without performing any reconstruction.

Firstly, we assume an infinitely large spot and a homogeneous luminescence source $S(x) \equiv S_{0}$. Secondly, we consider a very specific variation of transition energies in the form $\tilde{E}(u)=E_{0}+\sin (2 \pi u)$. While a real disorder potential in general is more randomly fluctuating and might even contain several characteristic length scales, our more regular model potential has a perfectly well defined length scale.

With the above mentioned approximations, we can simplify Eq. (10) for $\Omega=E_{0}$ and get

$$
\tilde{U}_{\Omega=E_{0}}(Q)=\int g_{\alpha}(\sin (2 \pi u)) e^{i Q u} \mathrm{~d} u
$$

After rewriting the broadened Lorentzian function as

$g_{\alpha}(\sin (2 \pi u))=\frac{i}{2 \pi}\left(\frac{1}{\sin (2 \pi u)+i \alpha}-\frac{1}{\sin (2 \pi u)-i \alpha}\right)$

we can solve the integral in Eq. (15) with the help of Cauchy's theorem where the contour of the integration has to be closed in the upper (lower) complex plane for positive (negative) sign of $Q$, respectively.

The poles of the two contributions of Eq. (16) are given by the roots of the energy denominators. The roots of the first denominator are defined by the equation

$$
\sin (2 \pi u)=-i \alpha
$$

Introducing the quantity

$$
y_{0}=\operatorname{asinh}(\alpha)=\ln \left(\alpha+\sqrt{1+\alpha^{2}}\right),
$$

we can express the solutions of Eq. (17) as

$$
u_{1,-}^{(m)}=-\frac{i}{2 \pi} y_{0}+m, \quad u_{1,+}^{(m)}=+\frac{i}{2 \pi} y_{0}+m+\frac{1}{2},
$$

for integer values of $m$. The subscripts + and signify whether the pole lies in the upper or lower 
complex plane. Similarly, the poles of the second contribution of Eq. (16) are given by

$$
u_{2,-}^{(m)}=-\frac{i}{2 \pi} y_{0}+m+\frac{1}{2}, \quad u_{2,+}^{(m)}=+\frac{i}{2 \pi} y_{0}+m .
$$
by

The residues of the integrand of Eq. (15) are given

$$
\begin{aligned}
& \operatorname{Res}\left[\frac{i}{2 \pi} \frac{e^{i Q u}}{\sin (2 \pi u)+i \alpha} ; u_{1, \pm}^{(m)}\right]= \pm \frac{1}{2 \pi i} \frac{1}{2 \pi} \frac{e^{i Q u_{1, \pm}^{(m)}}}{\cosh \left(y_{0}\right)}, \\
& \operatorname{Res}\left[\frac{i}{2 \pi} \frac{e^{i Q u}}{\sin (2 \pi u)-i \alpha} ; u_{2, \pm}^{(m)}\right]=\mp \frac{1}{2 \pi i} \frac{1}{2 \pi} \frac{e^{i Q u_{2, \pm}^{(m)}}}{\cosh \left(y_{0}\right)},
\end{aligned}
$$

such that after inserting the explicit solutions for $u_{1 / 2, \pm}^{(m)}$ we obtain

$$
\tilde{U}_{\Omega=E_{0}}(Q)=\frac{1}{2 \pi} \frac{\mathrm{e}^{-\frac{|Q| y_{0}}{2 \pi}}}{\cosh \left(y_{0}\right)} \sum_{n=-\infty}^{\infty} \mathrm{e}^{i Q n / 2} .
$$

Employing the identity

$$
\sum_{n=-\infty}^{\infty} e^{i Q n / 2}=4 \pi \sum_{m=-\infty}^{\infty} \delta(Q-4 \pi m)
$$

we obtain the final result

$$
\tilde{U}_{\Omega=E_{0}}(Q)=4 \frac{g^{-\frac{|Q|}{2 \pi}}}{g+g^{-1}} \sum_{m=-\infty}^{\infty} \delta(Q-4 \pi m)
$$

Here we have introduced

$$
g=\alpha+\sqrt{1+\alpha^{2}}
$$

and used the definition of $y_{0}$ as well as the relations

$$
\begin{aligned}
\mathrm{e}^{-\frac{|Q| y_{0}}{2 \pi}} & =\left(\cosh \left(y_{0}\right)+\sinh \left(y_{0}\right)\right)^{-\frac{|Q|}{2 \pi}}=g^{-\frac{|Q|}{2 \pi}(27)} \\
\cosh \left(y_{0}\right) & =\sqrt{1+\alpha^{2}}=\frac{1}{2}\left(g+g^{-1}\right)
\end{aligned}
$$

Our result, Eq. (25), nicely demonstrates that for the sinusoidal model potential the expected angular correlation consists of regularly spaced delta functions multiplied with an envelop function which exponentially decays with $|Q|$. The decay rate with respect to $|Q|$ is determined by $\ln (g)$ and thus a function of the ratio $\alpha=\gamma / W$. The different peaks are located at integer multiples of $4 \pi$ corresponding to a spacing of $4 \pi / L$ for the unscaled variable $\Delta q$.

In order to confirm our results and study the influence of a finite spot size, we display the envelope function $4 g^{-\frac{|Q|}{2 \pi}} /\left(g+g^{-1}\right)$ together with the results of two numerical computations for finite spot size in Fig. 2a. Here, we have normalized the result to a maximum value of 1 and plotted the correlation as a function of $\Delta q / q_{0}$ where the wavenumber $q_{0}$ corresponds to an assumed emission wavelength of
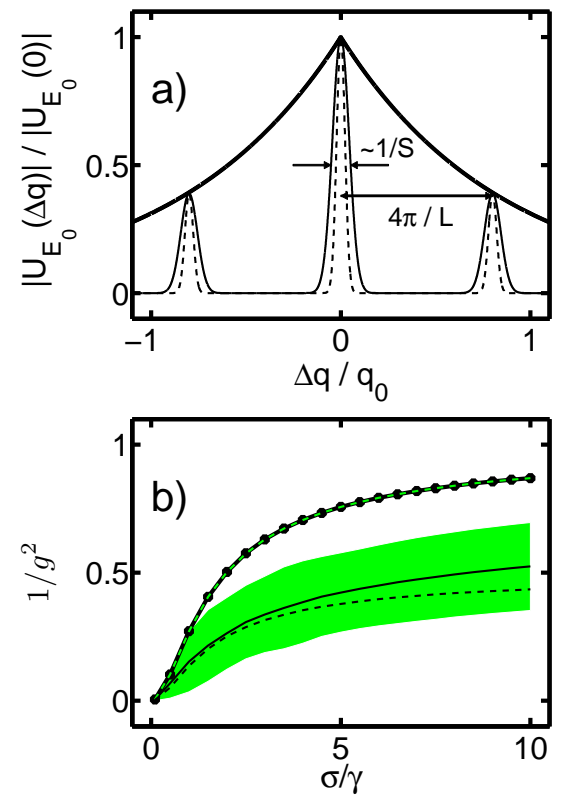

FIG. 2: a) Correlation function $U_{\hbar \omega=E_{0}}(\Delta q)$ as function of $\Delta q / q_{0}$ for a sinusoidal potential and different spotsizes. Comparison between the analytically computed envelope function (thick solid line) and the numerical result for a spotsize of $S=10 \mu \mathrm{m}$ (thin solid line) and $S=20 \mu \mathrm{m}$ (dashed line). Furthermore indicated are the peakwidth proportional to the inverse spotsize and the peak distance inversely proportaion to the disorder length scale. Numerical parameters are $\gamma=1 \mathrm{meV}$, $W / \gamma=20, L=1 \mu \mathrm{m}$.

b) The height of the second maximum of $U_{\hbar \omega=E_{0}}(\Delta q)$ as a function of the variance of the disorder potential. Thick line: sinusoidal potential, analytical result. Dots: numerical results for arbitrary spot size. The dashed line gives the thick line divided by 2 . For random potentials of various length scales $L$ the resulting curves are situated in the shaded area. The thin line is a guide for the eye.

$\lambda_{0}=800 \mathrm{~nm}$. The distance between neighbouring sites has been chosen $a=5 \mathrm{~nm}$.

We notice that our predictions are confirmed by the numerical simulations and that furthermore the finite spotsize does not influence the envelope function. While the width of the peaks is inversely proportional to the spotsize, both their height and their position do not depend on $\mathcal{S}$. In a real experiment, we can thus expect that the distance between the central peak and the first neighbouring peak is a signature of the longest disorder length scale involved, while the ratio between the peak heights of first and second peak, given by

$$
\frac{\tilde{U}_{\Omega=E_{0}}(4 \pi)}{\tilde{U}_{\Omega=E_{0}}(0)}=g^{-2},
$$


can provide a measure of the ratio between homogeneous $\gamma$ and the disorder fluctuation $W$. For known homogeneous broadening, one can thus extract information on the energetic spread of the transition energies.

A good measure of the energy fluctuations for very different kinds of disorder potentials is given by the standard deviation $\sigma$. For a general random potential used in a numerical computation, the standard deviation is obtained via

$$
\sigma^{2}=\frac{1}{N} \sum_{j}\left(E_{j}-\bar{E}\right)^{2}
$$

as a sum over the lattice sites, where $\bar{E}$ is the average energy and $N$ is the total number of sites. For our sinusoidal model potential in the continuum limit, we must use the integral form

$$
\sigma^{2}=\frac{1}{L} \int_{0}^{L} \sin ^{2}(2 \pi x / L) \mathrm{d} x=W^{2} / 2 .
$$

The result shows that the standard deviation in that case is simply proportional to the amplitude of the sinusoidal function. For a comparison between the analytical model system and numerical solutions with random disorder potentials, a systematic study of the ratio $g^{-2}$ as function of $\sigma / \gamma$ is shown in Fig. 2b. As a consequence of the random disorder potential, also the peak ratios for different realizations are different. The shaded area denotes the region in which all numerical results of a large number of simulations have been found. The analytical result (thick solid line) is found to be roughly twice as large over the whole range of $\sigma$ values. Analytical tests with a superposition of more than one sine function have shown that the analytical result for such a model also decreases towards the numerically observed region.

\section{CONCLUSIONS}

In conclusion, the presented analytical derivation of angular photonic correlations supports the results of previously published numerical investigations [11] and helps to understand the underlying principles of the suggested reconstruction scheme.

We have derived the angular correlation function for an ensemble of uncoupled two-level systems. In that model, the correlation function has been found to be given by the Fourier transform of the excitation spot for a perfectly ordered system while the presence of disorder shows up in a deviation from this shape. The spatial distribution of emitters can be recovered via a Fourier transformation. For a sinusoidal model potential analytical formulas have been derived for the dependency of the primary experimentally detectable signal on the characteristic strength and length scale of the disorder potential.

\section{Acknowledgments}

The authors are grateful to H. Stolz, K. Maschke, and T. Meier for valuable discussions. P. B. and H. S. gratefully acknowledge the financial support by the European Commission, Marie Curie Excellence Grant MEXT-CT-2005-023778 (Nanoelectrophotonics). In Marburg, this work has been supported by the Optodynamics Center of the Philipps-University Marburg and by the Deutsche Forschungsgemeinschaft through the Quantum Optics in Semiconductors Research Group. I.V. thanks for financial support from OTKA (Hungarian Research Fund) under Contracts No. T042981 and No. T46303.
[1] H. Overhof and P. Thomas, "Electronic Transport in Hydrogenated Amorphous Semiconductors", Springer Tracts in Mod. Phys., Vol. 114 (Springer, Berlin, 1989)

[2] B. I. Shklovskii and A. L. Efros, "Electronic Properties of Doped Semiconductors" (Springer 1984).

[3] "Optical Properties of Mixed Crystals", Ed. R. J. Elliott and I. P. Ipatova (Elsevier, 1988)

[4] T. Meier, P. Thomas, and S. W. Koch, "Coherent Semiconductor Optics: From Basic Concepts to Nanostructure Applications" (Springer-Verlag, 2007)

[5] Y. Yayon et.al., Phys. Rev. Lett. 89, 157402 (2002)

[6] C. Ell et.al., Phys. Rev. Lett. 80, 4795 (1998)

[7] A. V. Shchegrov et.al., Phys. Rev. Lett. 84, 3478 (2000)

[8] S. T. Bramwell, Nature, 439, 19 (2006)

[9] I. V. Gornyi et.al., Phys. Rev. Lett. 95, 206603
(2005)

[10] W. Langbein et.al., Phys. Rev. Lett. 89, 157401 (2002)

[11] P. Bozsoki et.al., Phys. Rev. Letters 97, 227402 (2006), arXiv: cond-mat/0611411.

[12] U. Neubert et al., Appl. Phys. Lett. 80, 3340 (2002)

[13] G. von Freymann et al., Phys. Rev. B65, 205327 (2002)

[14] A. Richter et al., Phys. Rev. Lett. 79, 2145 (1997)

[15] R. Cingolani et al., J. of Appl. Phys. 86, 6793 (1999)

[16] M. Kira et.al., Progress in Quantum Electronic, 23, Number 6 (1999)

[17] P. Bozsoki et.al., J. Lumin. 124, 99 (2007), arXiv: cond-mat/0505207.

[18] W. Hoyer et.al., Phys. Rev. Letters 93, 067401 (2004). 